\title{
A systematic review and meta-analysis of minimally invasive vs. standard percutaneous nephrolithotomy in the surgical management of renal stones
}

\author{
BINBIN JIAO ${ }^{1,2^{*}}$, ZHENKAI LUO $^{1,3^{*}}$, TAO HUANG ${ }^{1,3}$, GUAN ZHANG $^{1-3}$ and JIANG YU ${ }^{4}$ \\ ${ }^{1}$ Department of Urology, China-Japan Friendship Hospital, Beijing 100029; ${ }^{2}$ Graduate School, Peking \\ Union Medical College and Chinese Academy of Medical Sciences, Beijing 100730; ${ }^{3}$ Medical College, Peking University \\ China-Japan Friendship School of Clinical Medicine, Beijing 100029; ${ }^{4}$ Department of Urology, \\ Shandong Provincial Hospital Affiliated to Shandong First Medical University, Jinan, Shandong 250021, P.R. China
}

Received December 23, 2019; Accepted June 24, 2020

DOI: 10.3892/etm.2021.9645

\begin{abstract}
The present study aimed to assess current evidence on the effectiveness and safety of minimally invasive vs. standard percutaneous nephrolithotomy (PCNL) in the management of renal stones. A systematic search of electronic databases, which included PubMed, EMBASE and the Cochrane Library up to May 2019 was performed. Using Review Manager statistical software (version 5.3), primary outcomes, including stone-free rates (SFRs), were evaluated. Meanwhile, analysis was also performed to compare secondary outcomes, such as peri- and postoperative complications and operative data. Fourteen studies involving 1,611 patients with renal stones were analyzed based on the inclusion criteria. On the basis of the present analysis, mini percutaneous nephrolithotomy (MPCNL) was proven to have non-inferior clinical efficacy with respect to the SFR compared with PCNL [odds ratio $(O R)=1.10 ; 95 \%$ confidence interval $(C I), 0.84-1.44 ; \mathrm{P}=0.48]$. In addition, the meta-analysis showed that MPCNL had a significantly lower hemoglobin decrease [mean difference $(\mathrm{MD})=-0.68 ; 95 \% \mathrm{CI},-1.05$ to $-0.31 ; \mathrm{P}=0.0003$ ] and fewer blood transfusions $(\mathrm{OR}=0.36 ; 95 \% \mathrm{CI}, 0.18-0.71 ; \mathrm{P}=0.003)$ compared with PCNL. Moreover, the MPCNL group had
\end{abstract}

Correspondence to: Professor Guan Zhang, Department of Urology, China-Japan Friendship Hospital, 2 Yinghuadong Road, Beijing 100029, P.R. China

E-mail: gzhang2016@sina.com

Professor Jiang Yu, Department of Urology, Shandong Provincial Hospital Affiliated to Shandong First Medical University, 325 Five Weft Seven Road, Jinan, Shandong 250021, P.R. China

E-mail:dr.mrna@163.com

${ }^{*}$ Contributed equally

Key words: percutaneous nephrolithotomy, minimally invasive percutaneous nephrolithotomy, renal stones, mini percutaneous nephrolithotomy a shorter inpatient stay $(\mathrm{MD}=-0.81 ; 95 \% \mathrm{CI},-1.55$ to -0.08 ; $\mathrm{P}=0.03$ ) compared with the PCNL group. However, the overall evidence was insufficient to suggest a statistically significant difference in the adverse event profile for MPCNL compared with PCNL. The present meta-analysis indicates that MPCNL is an effective method for treating renal stones. Compared with PCNL, MPCNL not only has similarly high SFRs but is also associated with less blood loss, fewer blood transfusions, more favorable recovery time and shorter inpatient stays. However, the findings of the present study should be further confirmed by well-designed prospective randomized controlled trials with a larger patient series.

\section{Introduction}

Globally, kidney stone formation is one of the three most common diseases in urology, accounting for $80-90 \%$ of all urinary calculi $(1,2)$. Percutaneous nephrolithotomy (PCNL) is the standard treatment for renal stones $>2 \mathrm{~mm}$ and is a suitable choice for smaller renal stones (3). Although this approach has high stone-free rates (SFRs), it is associated with more postoperative complications, such as postoperative bleeding, fever and postoperative pain, compared with other approaches, such as extracorporeal shockwave lithotripsy and retrograde intrarenal surgery $(4,5)$. The most important disadvantage of PCNL is that it is associated with increased complications related to larger tract access (6). In an effort to reduce complications related to PCNL or to make the procedure more suitable for children, minimally invasive PCNL (MPCN), which uses a smaller tract, was introduced to treat urinary stones $(7,8)$. MPCNL is a modified PCNL technique with a miniaturized scope that moves through a smaller $(\leq 22 \mathrm{~F})$ nephrostomy tract (9). Although MPCNL was more likely to be associated with fewer complications, there was still controversy about its relative efficiency in the clearance of stones compared with standard PCNL, as the smaller tract may hinder the required instrumental manipulations for stone removal, especially for large calculi (10). In recent years, an increasing number of studies have been conducted to assess the clinical efficiency, operative results and complications of MPCNL and PCNL, 
but the outcomes of these studies have varied (11-13). Thus, it is worth conducting a new systematic review and meta-analysis that includes the relevant, available randomized controlled trials (RCTs) and prospective studies to date to evaluate the efficacy of MPCNL and PCNL.

\section{Materials and methods}

Search strategy. To assess the clinical efficacy and safety of MPCNL and PCNL, a comprehensive literature search was performed using PubMed (https://pubmed.ncbi.nlm.nih.gov/), Embase (https://www.embase.com/) and the Cochrane Library (https://www.cochranelibrary.com/) between March 2019 and May 2019. The keywords 'mini percutaneous nephrolithotomy', 'percutaneous nephrolithotomy', 'renal stone', 'minimally invasive' and 'standard' were used to search for articles. These search terms were used individually and in combination. Additionally, hand searches were performed for the references and citation lists of all relevant reviews. For publication selection, a search strategy was applied based on the Preferred Reporting Items for Systematic Reviews and Meta-analysis statement (14) and the Assessing the Methodological Quality of Systematic Reviews guidelines (15). Only RCTs and prospective studies comparing MPCNL with PCNL were included. Relevant references cited in the selected papers were also retrieved. No language restrictions were applied and the search was limited to human studies. Literature search, selection and data extraction were independently performed by two reviewers (ZL and BJ) and were then cross-checked. Any differences at this stage were resolved through discussion and if necessary, by a majority decision of the reviewers. A flowchart showing the number of publications selected or excluded at each stage is presented in Fig. 1. Ethical Committee approval for the current study was not required as all data were carefully extracted from existing literature and this article did not involve the handling of individual patient data.

Assessment of study quality. The levels of evidence for each selected article were evaluated based on the criteria recommended by the Oxford Centre for Evidence-based Medicine (16). For methodological quality assessment, the Jadad scale (17) was used to assess the quality of RCTs and the Newcastle-Ottawa Scale (18) was used to evaluate the quality of prospective studies (Table I). In addition, the Cochrane Collaboration tool (19) was used to assess the risk of bias of RCTs. Twelve relevant studies $(12,20-30)$ which included 1,611 patients were selected for analysis (Table II). No differences were found in terms of age and basic physical conditions between the MPCNL and PCNL groups. Data were independently extracted by two authors (BJ and ZL). The mean difference (MD) or standardized mean difference (SMD) was used to evaluate continuous outcomes. For studies expressing continuous data as the median and range values, the statistical formula described by Hozo et al (31) was used to determine the mean and standard deviation. The results are expressed as the risk ratio (RR) with a $95 \%$ confidence interval (CI) for dichotomous variables. The $\chi^{2}$ and $\mathrm{I}^{2}$ tests $\left(\mathrm{I}^{2}>50 \%\right.$ was regarded as substantial heterogeneity) were used to assess the heterogeneity of the study data. A random-effects model was used to reduce the effect of statistical heterogeneity for the meta-analyses. The pooled effects were determined by a $\mathrm{z}$ test and a P-value $<0.05$ was considered to indicate a statistically significant difference. Moreover, for comparisons between MPCNL and PCNL, relevant publications with appropriate data was used to perform subgroup analyses according to the device used. For several comparisons, sensitivity analyses were used. The meta-analysis of comparable data was performed using Review Manager software (version 5.3; Cochrane).

\section{Results}

Quality assessment. Among seven RCTs, most studies had a low risk of bias. Considering the ethical factor of surgery, it is not ideal to achieve blind methods. Therefore, four trails (20,28-30) were evaluated to have high risk for blinding of participants and personnel according to the article content (Fig. 2).

Overall SFR. Twelve studies involving 1,611 participants were included that compared the SFRs of MPCNL and PCNL. The overall results showed that the SFRs of the two groups were not significantly different [odd ratio $(\mathrm{OR})=1.10 ; 95 \% \mathrm{CI}$, 0.84-1.44; P=0.48; Fig. 3]

SFR according to size. Five studies compared the SFRs for calculi $>2 \mathrm{~cm}$ and the meta-analysis showed no significant difference between the groups $(\mathrm{OR}=1.06$; 95\% CI, 0.64-1.74; $\mathrm{P}=0.82$; Fig. 4A). Two studies compared the SFR for calculi $<2 \mathrm{~cm}$ and the SFR was not significantly different between MPCNL and PCNL groups (OR=0.54; 95\% CI, 0.11-2.70; $\mathrm{P}=0.45$; Fig. 4B).

SFR of staghorn stones. Two studies reported the SFR in calculi that were classified as staghorn stones. There was no significant difference between the two groups $(\mathrm{R} R=1.11$; 95\% CI, 0.87-1.42; P=0.40; Fig. 4C).

SFR according to follow-up time. For SFRs within 1 week, this rare outcome was reported in three trials that compared MPCNL and PCNL. When pooled, the results showed that there was no significant difference between the two groups ( $R R=1.03$; 95\% CI, 0.96-1.10; $\mathrm{P}=0.41$; Fig. 4D). For SFRs at 1 month, four trials that included 417 participants were included in the present analysis. The present results verified that there was no significant difference between the two groups $(\mathrm{OR}=1.02$; 95\% CI, 0.53-1.99; $\mathrm{P}=0.94$; Fig. 4E).

Operation duration. Twelve trials that met the inclusion criteria that compared MPCNL to PCNL and the overall operation time was approximately $7 \mathrm{~min}$ less for PCNL compared with MPCNL (MD=7.62; 95\% CI, 1.05-14.19; P=0.02; Fig. 5A).

Operation time of staghorn stones. Regarding the operation time of staghorn stones, two studies were included in the meta-analysis. The results demonstrated that PCNL incurred a shorter time compared with MPCNL $(\mathrm{MD}=20.83$; 95\% CI, 7.11-34.54; $\mathrm{P}=0.003$; Fig. 5B).

Operative hemoglobin decrease. Eight studies that reported the operative hemoglobin decrease and compared MPCNL to PCNL were included in the meta-analysis. While the sensitivity analysis suggested that this factor had little impact on the 
Table I. Summary of comparative studies included in the meta-analysis.

\begin{tabular}{|c|c|c|c|c|c|c|c|c|c|c|}
\hline \multirow[b]{2}{*}{ Author, year } & \multirow[b]{2}{*}{ Country } & \multirow[b]{2}{*}{ Study period } & \multirow[b]{2}{*}{ Study design } & \multirow[b]{2}{*}{ LE } & \multicolumn{2}{|c|}{ Intervention } & \multicolumn{2}{|c|}{$\begin{array}{l}\text { Sample } \\
\text { size (n) }\end{array}$} & \multirow{2}{*}{$\begin{array}{l}\text { Study } \\
\text { quality }\end{array}$} & \multirow[b]{2}{*}{ (Refs.) } \\
\hline & & & & & Trial & Control & Trial & Control & & \\
\hline Du et al, 2018 & China & 2009-2014 & $\mathrm{RCT}$ & $2 b$ & MPCNL & PCNL & 304 & 297 & $3^{\mathrm{a}}$ & $(20)$ \\
\hline Güler et al, 2019 & Turkey & 2009-2011 & $\mathrm{RCT}$ & $2 \mathrm{a}$ & MPCNL & PCNL & 51 & 46 & $4^{\mathrm{a}}$ & $(21)$ \\
\hline Haghighi et al, 2017 & Iran & 2016-2017 & $\mathrm{RCT}$ & $2 \mathrm{a}$ & MPCNL & PCNL & 35 & 35 & $3^{\mathrm{a}}$ & $(22)$ \\
\hline Karakan et al, 2017 & Turkey & 2014-2016 & RCT & $2 \mathrm{a}$ & MPCNL & PCNL & 47 & 50 & $3^{\mathrm{a}}$ & (23) \\
\hline Knoll et al, 2010 & Germany & N/A & Prospective study & $3 b$ & MPCNL & PCNL & 25 & 25 & $6^{\mathrm{b}}$ & (24) \\
\hline Kukreja et al, 2018 & India & 2015-2017 & Prospective study & $2 b$ & MPCNL & PCNL & 61 & 62 & $7^{\mathrm{b}}$ & $(25)$ \\
\hline Li et al, 2010 & China & $2005-2008$ & Prospective study & $3 b$ & MPCNL & PCNL & 93 & 72 & $6^{\mathrm{b}}$ & (26) \\
\hline Mishra et al, 2011 & India & $2009-2010$ & Prospective study & $3 b$ & MPCNL & PCNL & 27 & 28 & $9^{\mathrm{b}}$ & $(27)$ \\
\hline Sakr et al, 2017 & Egypt & $2010-2013$ & $\mathrm{RCT}$ & $2 \mathrm{a}$ & MPCNL & PCNL & 87 & 81 & $3^{\mathrm{a}}$ & (28) \\
\hline Song et al, 2011 & China & 2008-2009 & $\mathrm{RCT}$ & $2 b$ & MPCNL & PCNL & 30 & 30 & $2^{\mathrm{a}}$ & (29) \\
\hline Xu et al, 2014 & China & 2011-1013 & Prospective study & $3 b$ & MPCNL & PCNL & 37 & 34 & $6^{\mathrm{b}}$ & (12) \\
\hline Zhong et al, 2011 & China & 2008-2009 & $\mathrm{RCT}$ & $2 b$ & MPCNL & PCNL & 29 & 25 & $3^{\mathrm{a}}$ & (30) \\
\hline
\end{tabular}

a Jadad scale (score, 0-5); b Newcastle-Ottawa Scale (score, 0-9). LE, level of evidence; Ref., reference; RCT, randomized controlled trial; PCNL, percutaneous nephrolithotomy; MCPNL, minimally invasive PCNL; N/A, not applicable.

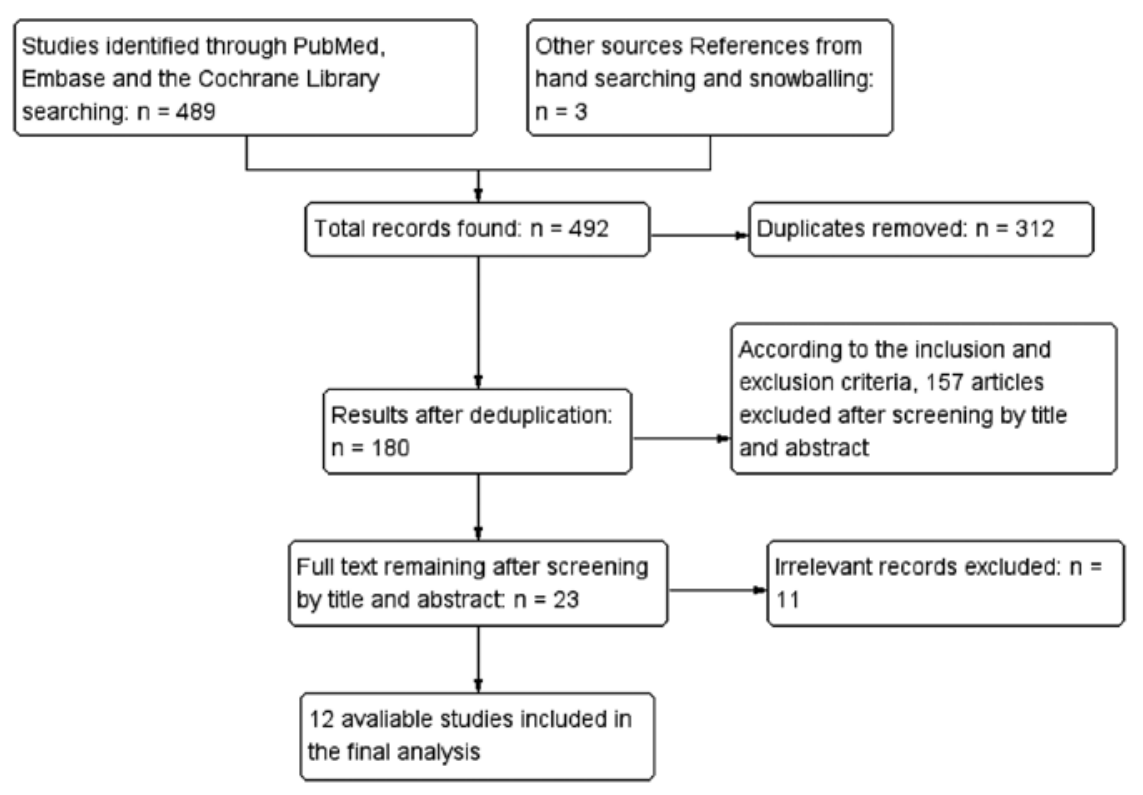

Figure 1. PRISMA flowchart.

combined result, a significantly lower operative hemoglobin decrease was observed for MPCNL compared with PCNL (MD=-0.68; 95\% CI, -1.05 to $-0.31 ; \mathrm{P}=0.0003$; Fig. 6).

Blood transfusion. Eleven studies involving 1,530 patients compared the blood transfusions between the two groups. The meta-analysis demonstrated that there were fewer blood transfusions in the MPCNL group compared with the PCNL group $(\mathrm{OR}=0.36 ; 95 \% \mathrm{CI}, 0.18-0.71 ; \mathrm{P}=0.003$; Fig. 7).

Hospitalization time. Regarding the length of inpatient stays, eight studies were included in the meta-analysis. When pooled, the results showed that the MPCNL group had a significantly shorter hospitalization time than the PCNL group (MD=-0.81; $95 \%$ CI, -1.55 to -0.08 ; $\mathrm{P}=0.03$; Fig. 8).

Postoperative visual analogue scale (VAS). For the VAS, a total of four studies that included 393 participants met the inclusion criteria. The combined result did not show a significant difference between MPCNL and PCNL groups (MD=-0.62; 95\% CI, -1.44-0.19; P=0.13; Fig. 9).

Postoperative fever. To evaluate long-term postoperative fever, twelve studies were included in the statistical analysis. The 
Table II. Baseline characteristics of included studies.

\begin{tabular}{|c|c|c|c|c|c|c|c|c|}
\hline Author, year & Treatment & $\begin{array}{l}\text { Stone } \\
\text { size }\end{array}$ & $\begin{array}{l}\text { Access } \\
\text { sheath } \\
\text { size }(F)\end{array}$ & Dilator & $\begin{array}{l}\text { Nephroscope } \\
\text { size (F) }\end{array}$ & Lithotripsy & $\begin{array}{l}\text { Follow-up } \\
\text { (SFR) }\end{array}$ & (Refs.) \\
\hline \multirow[t]{2}{*}{ Du et al, 2018} & MPCNL & $\begin{array}{c}9.54 \pm 5.2 / \\
13.9 \pm 4.7 \mathrm{~cm}^{2}\end{array}$ & $16-18$ & FD & 12 & Laser & 3-5 days & (20) \\
\hline & PCNL & $12.4 \pm 6.4 \mathrm{~cm}^{2}$ & 24 & FD & N/A & Laser & & \\
\hline \multirow[t]{2}{*}{ Güler et al, 2019} & MPCNL & $38.7 \pm 13.1 \mathrm{~mm}$ & $16.5 / 20$ & $\begin{array}{l}\text { Amplatz } \\
\text { dilator }\end{array}$ & 12 & Laser & 1 day & $(21)$ \\
\hline & PCNL & $38.7 \pm 13.1 \mathrm{~mm}$ & 30 & $\begin{array}{l}\text { Balloon } \\
\text { dilator }\end{array}$ & 26 & $\begin{array}{l}\text { Pneumatic- } \\
\text { ultrasonic }\end{array}$ & & \\
\hline \multirow{2}{*}{$\begin{array}{l}\text { Haghighi et al, } \\
2017\end{array}$} & MPCNL & $14.26(5.3) \mathrm{mm}$ & 16 & FD & 9.8 & Pneumatic & $48 \mathrm{~h}$ & $(22)$ \\
\hline & PCNL & $15.35(5.85) \mathrm{mm}$ & 30 & $\begin{array}{l}\text { Amplatz } \\
\text { dilator }\end{array}$ & 24 & Pneumatic & & \\
\hline \multirow[t]{2}{*}{$\begin{array}{l}\text { Karakan et al, } \\
2017\end{array}$} & MPCNL & $20.3 \pm 3.0 \mathrm{~mm}$ & 14 & $\begin{array}{l}\text { Single shot } \\
14 \mathrm{~F} \text { dilator }\end{array}$ & $8 / 9.8$ & Laser & 1 month & (23) \\
\hline & PCNL & $20.9 \pm 3.6 \mathrm{~mm}$ & 24 & $\begin{array}{l}\text { Amplatz } \\
\text { dilator }\end{array}$ & $22-25$ & $\begin{array}{l}\text { Ultrasonic and } \\
\text { pneumatic }\end{array}$ & & \\
\hline \multirow[t]{2}{*}{ Knoll et al, 2010} & MPCNL & $18 \pm 3.3 \mathrm{~mm}$ & 18 & $\begin{array}{l}\text { Singlestep } \\
\text { metal dilators }\end{array}$ & N/A & Laser & 1 day & (24) \\
\hline & PCNL & $22 \pm 4.25 \mathrm{~mm}$ & 26 & Alken dilator & N/A & Ultrasound/laser & & \\
\hline Kukreja et al, 2018 & $\begin{array}{l}\text { MPCNL } \\
\text { PCNL }\end{array}$ & $\begin{array}{l}20.6 \pm 3.47 \mathrm{~mm} \\
21.5 \pm 3.53 \mathrm{~mm}\end{array}$ & $\begin{array}{c}16.5 / 17.5 \\
22 / 24\end{array}$ & $\begin{array}{l}\text { Teflon dilator } \\
\text { Teflon dilator }\end{array}$ & $\begin{array}{c}12 \\
20.5\end{array}$ & $\begin{array}{l}\text { Laser/pneumatic } \\
\text { Laser/pneumatic }\end{array}$ & 1 month & (25) \\
\hline Li et al, 2010 & $\begin{array}{l}\text { MPCNL } \\
\text { PCNL }\end{array}$ & $\begin{array}{l}28.6(9-78) \mathrm{mm} \\
30.4(12-8) \mathrm{mm}\end{array}$ & $\begin{array}{c}14-18 \\
30\end{array}$ & $\begin{array}{l}\text { FD } \\
\text { TMD }\end{array}$ & $\begin{array}{l}8 / 9.8 \\
\text { N/A }\end{array}$ & $\begin{array}{l}\text { Ultrasound/laser } \\
\text { Pneumatic }\end{array}$ & 1 day & (26) \\
\hline Mishra et al, 2011 & $\begin{array}{l}\text { MPCNL } \\
\text { PCNL }\end{array}$ & $\begin{array}{l}1.47 \pm 0.3 \mathrm{~cm}^{2} \\
1.49 \pm 0.6 \mathrm{~cm}^{2}\end{array}$ & $\begin{array}{l}14-18 \\
24-28\end{array}$ & $\begin{array}{l}\text { FD } \\
\text { TMD }\end{array}$ & $\begin{array}{c}\mathrm{N} / \mathrm{A} \\
12 / 14\end{array}$ & $\begin{array}{l}\text { Pneumatic } \\
\text { Pneumatic/ } \\
\text { laser/ultrasonic }\end{array}$ & 1 month & (27) \\
\hline Sakr et al, 2017 & $\begin{array}{l}\text { MPCNL } \\
\text { PCNL }\end{array}$ & $\begin{array}{l}2.7 \pm 0.2 \mathrm{~cm} \\
2.6 \pm 0.6 \mathrm{~cm}\end{array}$ & $\begin{array}{c}16.5 \\
30\end{array}$ & $\begin{array}{l}\text { TMD } \\
\text { TMD }\end{array}$ & $\begin{array}{l}12 \\
26\end{array}$ & $\begin{array}{l}\text { Pneumatic } \\
\text { Pneumatic }\end{array}$ & 1 day & (28) \\
\hline Song et al, 2011 & $\begin{array}{l}\text { MPCNL } \\
\text { PCNL }\end{array}$ & $\begin{array}{l}8.57 \pm 2.2 \mathrm{~cm}^{2} \\
8.65 \pm 2.0 \mathrm{~cm}^{2}\end{array}$ & $\begin{array}{c}16 \\
24 \mathrm{~F}\end{array}$ & $\begin{array}{l}\text { FD } \\
\text { TMD }\end{array}$ & $\begin{array}{l}\mathrm{N} / \mathrm{A} \\
24 \mathrm{~F}\end{array}$ & $\begin{array}{l}\text { Laser } \\
\text { Ultrasound + } \\
\text { pneumatic }\end{array}$ & 3-5 days & (29) \\
\hline Xu et al, 2014 & $\begin{array}{l}\text { MPCNL } \\
\text { PCNL }\end{array}$ & $\begin{array}{l}33.4 \pm 10.3 \mathrm{~mm} \\
41.4 \pm 10.9 \mathrm{~mm}\end{array}$ & $\begin{array}{l}16 \mathrm{~F} \\
24 \mathrm{~F}\end{array}$ & $\begin{array}{l}\text { FD } \\
\text { TMD }\end{array}$ & $\begin{array}{c}12 \mathrm{~F} \\
20.5 \mathrm{~F}\end{array}$ & $\begin{array}{l}\text { Laser } \\
\text { Ultrasound + } \\
\text { pneumatic }\end{array}$ & N/A & (12) \\
\hline Zhong et al, 2011 & $\begin{array}{l}\text { MPCNL } \\
\text { PCNL }\end{array}$ & $\begin{array}{l}11.7(8.8-22.8) \mathrm{cm}^{2} \\
10.8(8.4-20.2) \mathrm{cm}^{2}\end{array}$ & $\begin{array}{l}16 \mathrm{~F} \\
26 \mathrm{~F}\end{array}$ & $\begin{array}{l}\text { FD } \\
\text { FD }\end{array}$ & $\begin{array}{l}8 / 9.8 \mathrm{~F} \\
\mathrm{~N} / \mathrm{A}\end{array}$ & $\begin{array}{l}\text { Pneumatic } \\
\text { Pneumatic }\end{array}$ & 1 day & (30) \\
\hline
\end{tabular}

Data are presented as mean \pm standard deviation or mean (interquartile range). F, French units; SFR, stone-free rate; Ref., reference; PCNL, percutaneous nephrolithotomy; MCPNL, minimally invasive PCNL; N/A, not applicable; SD, standard deviation; IQR, interquartile range; FD, fascial dilators; TMD, telescoping metal dilators, N/A not available; F, French units; SFR, stone-free rate.

meta-analysis revealed that there was no significant difference in the incidence of repeated surgery between these two groups $(\mathrm{OR}=1.27$; 95\% CI, 0.89-1.82; $\mathrm{P}=0.19$; Fig. 10).

Urinary perforation. Urinary perforation outcomes were reported in five studies, all of which compared MPCNL to PCNL. A total of 16 events were reported among 429 participants. On the basis of the present analysis, no significant heterogeneity was found among these trials $\left(\mathrm{I}^{2}=0\right)$ and the overall result revealed that the MPCNL group was similar to the PCNL group in regard to this outcome $(\mathrm{OR}=0.96 ; 95 \% \mathrm{CI}$, 0.34-2.67; $\mathrm{P}=0.93$; Fig. 11A).

Urinary leakage. With regard to leakage, four studies comparing MPCNL to PCNL were included. A total of 13 events were reported among 371 participants. On the basis of 

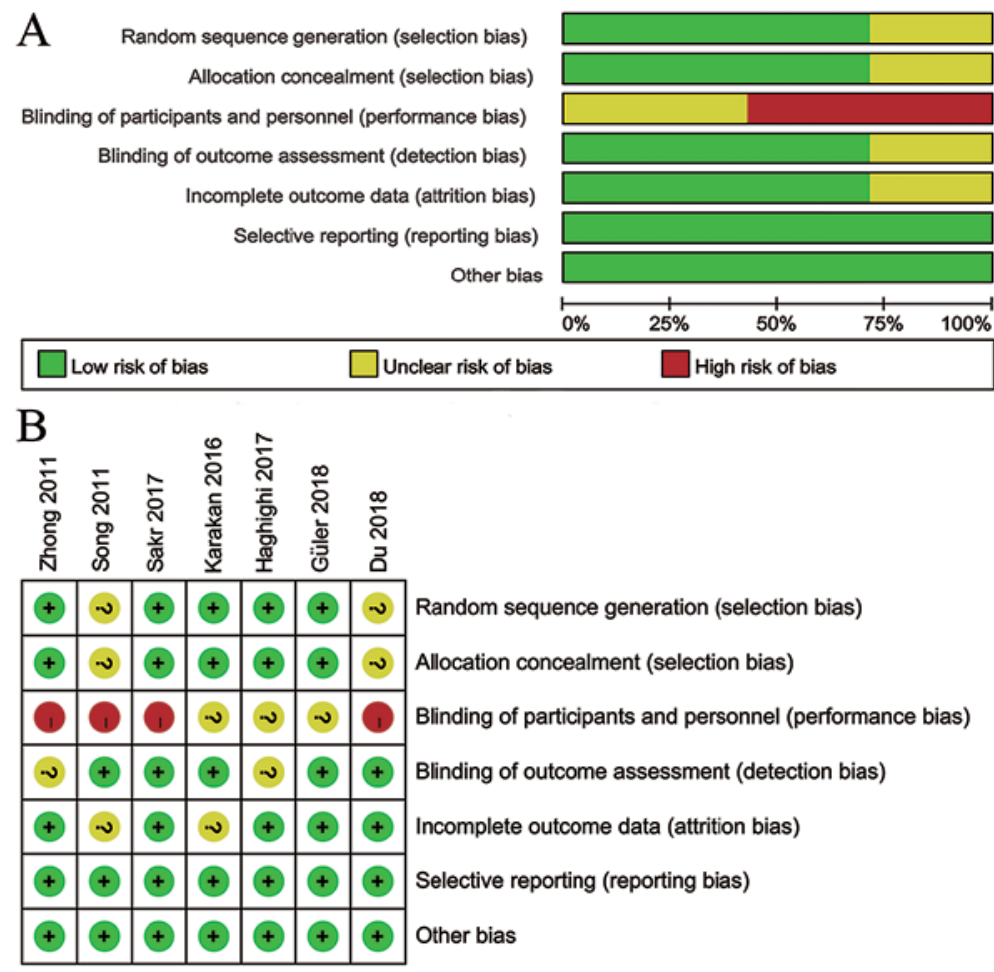

Figure 2. Risk of bias assessment using the Cochrane Collaboration tool. (A) Risk of bias graph. (B) Risk of bias summary. +, low risk of bias; ?, unclear risk of bias; -, high risk of bias.

\begin{tabular}{|c|c|c|c|c|c|c|c|c|c|c|}
\hline Study or Subgroup & $\begin{array}{l}\text { MPCNL } \\
\text { Events }\end{array}$ & $\begin{array}{l}\text { L } \\
\text { Total }\end{array}$ & $\begin{array}{l}\text { PCNL } \\
\text { Events }\end{array}$ & & Weight & $\begin{array}{c}\text { Odds Ratio } \\
\mathrm{M}-\mathrm{H} \text {. Random, } 95 \% \mathrm{Cl}\end{array}$ & & $\begin{array}{r}\text { Odds } \\
\text { M-H, Rand }\end{array}$ & $\begin{array}{l}\text { Ratio } \\
\text { lom. } 95 \% \mathrm{Cl}\end{array}$ & \\
\hline Du 2018 & 225 & 304 & 217 & 297 & $55.0 \%$ & $1.05[0.73,1.51]$ & & & & \\
\hline Güler 2018 & 39 & 51 & 33 & 46 & $8.7 \%$ & $1.28[0.51,3.18]$ & & & & \\
\hline Haghighi 2017 & 32 & 35 & 33 & 35 & $2.1 \%$ & $0.65[0.10,4.13]$ & & & & \\
\hline Karakan 2016 & 42 & 47 & 44 & 50 & $4.6 \%$ & $1.15[0.32,4.04]$ & & & & \\
\hline Knoll 2010 & 24 & 25 & 23 & 25 & $1.2 \%$ & $2.09[0.18,24.61]$ & & & & \\
\hline Kukreja2017 & 57 & 61 & 57 & 62 & $3.9 \%$ & $1.25[0.32,4.90]$ & & & & \\
\hline Li 2010 & 78 & 93 & 63 & 72 & $9.1 \%$ & $0.74[0.30,1.81]$ & & & & \\
\hline Mishra 2011 & 26 & 27 & 28 & 28 & $0.7 \%$ & $0.31[0.01,7.95]$ & & & & \\
\hline Sakr 2017 & 83 & 87 & 79 & 81 & $2.4 \%$ & $0.53[0.09,2.95]$ & & & & \\
\hline Song 2011 & 27 & 30 & 22 & 30 & $3.5 \%$ & $3.27[0.77,13.83]$ & & & & \\
\hline Xu 2014 & 29 & 37 & 27 & 34 & $5.5 \%$ & $0.94[0.30,2.94]$ & & & & \\
\hline Zhong 2011 & 26 & 29 & 17 & 25 & $3.4 \%$ & $4.08[0.95,17.58]$ & & & & \\
\hline Total $(95 \% \mathrm{Cl})$ & & 826 & & 785 & $100.0 \%$ & $1.10[0.84,1.44]$ & & & & \\
\hline Total events & 688 & & 643 & & & & & & & \\
\hline \multicolumn{7}{|c|}{$\begin{array}{l}\text { Heterogeneity: } \text { Tau }^{2}=0.00 ; \mathrm{Chi}^{2}=8.18, \mathrm{df}=11(P=0.70) ; \mathrm{I}^{2}=0 \% \\
\text { Test for overall effect: } Z=0.71(P=0.48)\end{array}$} & 0.02 & $\begin{array}{l}0.1 \\
\text { Favours [MPCNL] }\end{array}$ & $\begin{array}{l}10 \\
\text { Favours [PCNL] }\end{array}$ & 50 \\
\hline
\end{tabular}

Figure 3. Forest plot of the overall stone-free rate. Black diamonds indicate study weight. Blue squares indicate overall result. Horizontal lines indicate the 95\% confidence interval. CI, confidence interval; df, degrees of freedom; M-H, Mantel-Haenszel; Random, random effects model; SD, standard deviation; PCNL, percutaneous nephrolithotomy; MCPNL, minimally invasive PCNL.

the present analysis, no heterogeneity was found among the trials $\left(I^{2}=0\right)$. No significant differences were found between the two groups (RR=0.50; 95\% CI, 0.15-1.69; $\mathrm{P}=0.27$; Fig. 11B).

Delayed hemorrhage. Only two studies were available for meta-analysis regarding delayed hemorrhage. There was no significant difference between the two groups $(\mathrm{OR}=0.42$; 95\% CI, 0.06-2.95; P=0.38; Fig. 11C).

Complications. The incidence of Clavien-Dindo grade (32) I complications was not significantly different between the groups $(\mathrm{OR}=1.30 ; 95 \% \mathrm{CI}, 0.44-3.88 ; \mathrm{P}=0.64)$ and there was no difference in terms of the incidence of Clavien-Dindo grade II complications ( $\mathrm{OR}=0.49$; $95 \% \mathrm{CI}, 0.22-1.09$; $\mathrm{P}=0.08)$. In addition, the incidence of Clavien-Dindo Grade III complications was not significantly different between the groups $(\mathrm{OR}=0.67$; 95\% CI, 0.27-1.62; P=0.37; Fig. 12).

Sensitivity analysis and publication bias. For outcomes with high heterogeneity, sensitivity analysis was performed on a subgroup of RCTs (Table III). Most outcomes, including SFR, operative hemoglobin decrease and postoperative VAS, were not significantly different, indicating that the results of the present meta-analysis were stable. Meanwhile, significant differences in operation time and hospital stay between the two groups were not found due to the difference in patient 


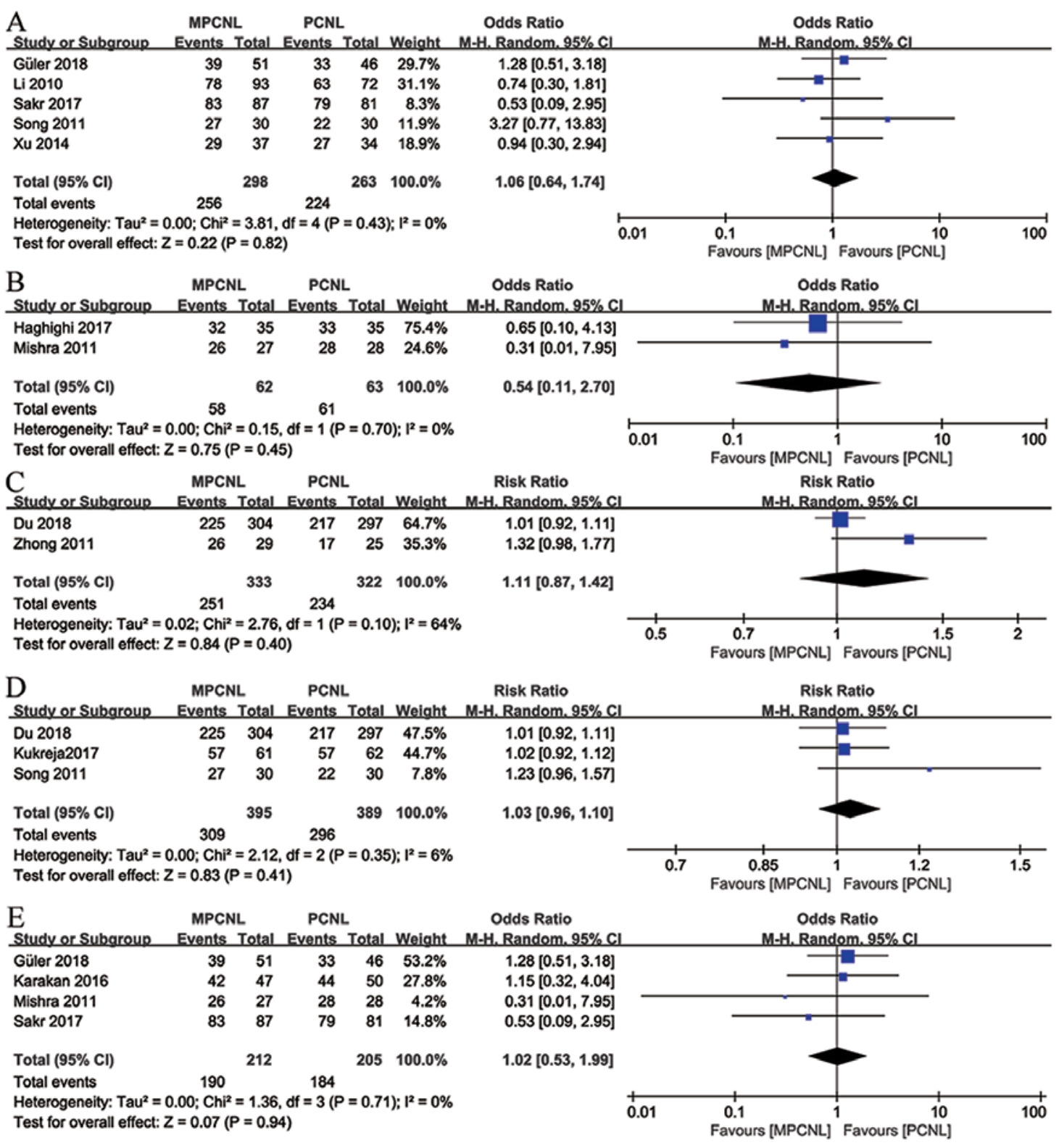

Figure 4. Forest plots of the stone-free rate subgroups. (A) SFR analysis of stones $>2 \mathrm{~cm}$. (B) SFR analysis of stones $<2 \mathrm{~cm}$. (C) SFR analysis of staghorn stones (D) SFR analysis within 1 week. (E) SFR analysis within 1 month. Black diamonds indicate study weight. Blue squares indicate overall result. Horizontal lines indicate the 95\% confidence interval. CI, confidence interval; df, degrees of freedom; M-H, Mantel-Haenszel; Random, random effects model; SD, standard deviation; PCNL, percutaneous nephrolithotomy; MCPNL, minimally invasive PCNL; SFR, stone-free rate.

inclusion criteria, outcome definitions and standards, surgical experience, follow-up imaging and duration. Funnel plots were used to assess the publication bias of included studies. The result showed no apparent asymmetry, which indicated no obvious publication bias (Fig. 13).

\section{Discussion}

Kidney calculi are a common urological disorder characterized by a high recurrence rate (33). PCNL is currently the gold standard treatment for large kidney stones $(3,34)$. Although PCNL had high SFRs, it is associated with complications such as bleeding and blood transfusion (35-37). In an effort to reduce morbidity related to PCNL instruments with large diameter channels (38), a renewed need for a definitive treatment for bulk urolithiasis and improvements in less invasive approaches have gained interest in other techniques. MPCNL utilizes a smaller tract dilation diameter and was introduced to reduce the complications associated with a larger access tract (37). To date, MPCNL has not been standardized, leading sheath diameters $<22 \mathrm{~F}$ to be defined as miniaturized (9). Although a number of studies have shown the effectiveness of PCNL and MPCNL for the treatment of renal stones, the results have been controversial $(24,26,28)$. The purpose of the present meta-analysis was to evaluate and compare the efficacy and safety of PCNL and MPCNL for the treatment of renal calculi.

The SFR is the most important parameter for estimating the efficacy of these two approaches (39). In theory, with respect to the surgical process, PCNL has a higher SFR compared with MPCNL because the larger access tract provides a wider view (40). In the present meta-analysis, the results revealed no 


\begin{tabular}{|c|c|c|c|c|c|c|c|c|c|c|}
\hline \multirow{2}{*}{$\begin{array}{l}\text { A } \\
\text { Study or Subgroup }\end{array}$} & \multicolumn{3}{|c|}{ MPCNL } & \multicolumn{3}{|c|}{ PCNL } & \multirow{2}{*}{\multicolumn{2}{|c|}{$\begin{array}{ll} & \text { Mean Difference } \\
\text { Weight } & \text { IV. Random, } 95 \% \mathrm{Cl}\end{array}$}} & \multirow{2}{*}{\multicolumn{2}{|c|}{$\begin{array}{l}\text { Mean Difference } \\
\text { IV. Random. } 95 \% \mathrm{Cl}\end{array}$}} \\
\hline & Mean & SD & Total & Mean & SD & Total & & & & \\
\hline Du 2018 & 81 & 41 & 304 & 53 & 27 & 297 & $9.0 \%$ & $28.00[22.46,33.54]$ & & \\
\hline Güler 2018 & 89.2 & 40.4 & 51 & 74.7 & 44.5 & 46 & $5.8 \%$ & $14.50[-2.48,31.48]$ & & \\
\hline Haghighi 2017 & 48 & 4.3 & 35 & 51 & 5.6 & 35 & $9.5 \%$ & $-3.00[-5.34,-0.66]$ & & \\
\hline Karakan 2016 & 552 & 23.5 & 47 & 70 & 32.7 & 50 & $7.5 \%$ & $-15.00[-26.28,-3.72]$ & & \\
\hline Knoll 2010 & 592 & 29.1 & 25 & 49 & 21.7 & 25 & $6.6 \%$ & $10.00[-4.23,24.23]$ & & \\
\hline Kukreja2017 & 25.46 & 11.9 & 61 & 24.68 & 12.45 & 62 & $9.2 \%$ & $0.78[-3.52,5.08]$ & & \\
\hline Li 2010 & 87.6 & 31.6 & 93 & 64.5 & 25.7 & 72 & $8.2 \%$ & $23.10[14.35,31.85]$ & & \\
\hline Mishra 2011 & 45.2 & 12.6 & 27 & 31 & 16.6 & 28 & $8.4 \%$ & $14.20[6.43,21.97]$ & & \\
\hline Sakr 2017 & 83.2 & 17.3 & 87 & 78.6 & 24.4 & 81 & $8.8 \%$ & $4.60[-1.84,11.04]$ & & \\
\hline Song 2011 & 39 & 10 & 30 & 42 & 8 & 30 & $9.1 \%$ & $-3.00[-7.58,1.58]$ & & \\
\hline Xu 2014 & 115.4 & 13.5 & 37 & 110.6 & 17 & 34 & $8.6 \%$ & $4.80[-2.38,11.98]$ & & \\
\hline Zhong 2011 & 115.3 & 8.4 & 29 & 101.3 & 4.3 & 25 & $9.3 \%$ & $14.00[10.51,17.49]$ & & \\
\hline Total $(95 \% \mathrm{CI})$ & & & 826 & & & 785 & $100.0 \%$ & $7.62[1.05,14.19]$ & & \\
\hline \multicolumn{9}{|c|}{$\begin{array}{l}\text { Heterogeneity: } \text { Tau }^{2}=117.34 ; \mathrm{Ch}^{2}=186.99, \mathrm{df}=11(P<0.00001) ; \mathrm{I}^{2}=94 \% \\
\text { Test for overall effect: } Z=2.27(P=0.02)\end{array}$} & $\begin{array}{ccc}-50 & -25 & 0 \\
\text { Favours [MPCNL] }\end{array}$ & \begin{tabular}{|ccc}
0 & 25 & 50 \\
Favours [PCNL]
\end{tabular} \\
\hline $\begin{array}{l}\text { B } \\
\text { Study or Subgroup }\end{array}$ & $\begin{array}{c}\text { MF } \\
\text { Mean }\end{array}$ & $\begin{array}{l}\text { IPCNL } \\
\text { SD }\end{array}$ & Total & $\begin{array}{c}\mathrm{PC} \\
\text { Mean }\end{array}$ & $\begin{array}{l}\text { CNL } \\
\text { SD }\end{array}$ & Total & Weight & $\begin{array}{l}\text { Mean Difference } \\
\text { IV. Random, } 95 \% \mathrm{Cl}\end{array}$ & \multicolumn{2}{|c|}{$\begin{array}{l}\text { Mean Difference } \\
\text { IV. Random. } 95 \% \text { Cl }\end{array}$} \\
\hline Du 2018 & 81 & 41 & 304 & 53 & 27 & 297 & $48.8 \%$ & $28.00[22.46,33.54]$ & & 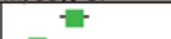 \\
\hline Zhong 2011 & 115.3 & 8.4 & 29 & 101.3 & 4.3 & 25 & $51.2 \%$ & $14.00[10.51,17.49]$ & & $\square$ \\
\hline Total $(95 \% \mathrm{Cl})$ & & & 333 & & & 322 & $100.0 \%$ & $20.83[7.11,34.54]$ & & \\
\hline \multicolumn{9}{|c|}{$\begin{array}{l}\text { Heterogeneity: } \text { Tau }^{2}=92.42 ; \mathrm{Chi}^{2}=17.57, \mathrm{df}=1(P<0.0001) ;\left.\right|^{2}=94 \% \\
\text { Test for overall effect: } Z=2.98(P=0.003)\end{array}$} & $\begin{array}{ccc}-50 & -25 & 0 \\
\text { Favours } & {[\mathrm{MPCNL}]}\end{array}$ & $\begin{array}{cc}25 & 50 \\
\text { Favours }[\mathrm{PCNL}\end{array}$ \\
\hline
\end{tabular}

Figure 5. Forest plots of the operative time. (A) Operation duration. (B) Operation time of staghorn stones. Black diamonds indicate study weight. Blue squares indicate overall result. Horizontal lines indicate the $95 \%$ confidence interval. CI, confidence interval; df, degrees of freedom; Random, random effects model; SD, standard deviation; PCNL, percutaneous nephrolithotomy; MCPNL, minimally invasive PCNL; IV, inverse variance.

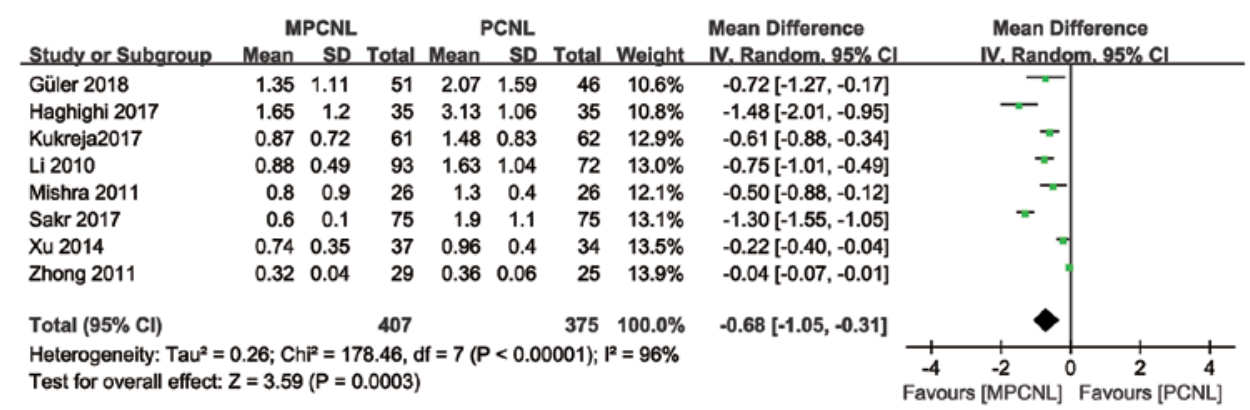

Figure 6. Forest plot of hemoglobin decrease. Black diamonds indicate study weight. Blue squares indicate overall result. Horizontal lines indicate the 95\% confidence interval. CI, confidence interval; df, degrees of freedom; Random, random effects model; SD, standard deviation; PCNL, percutaneous nephrolithotomy; MCPNL, minimally invasive PCNL; IV, inverse variance.

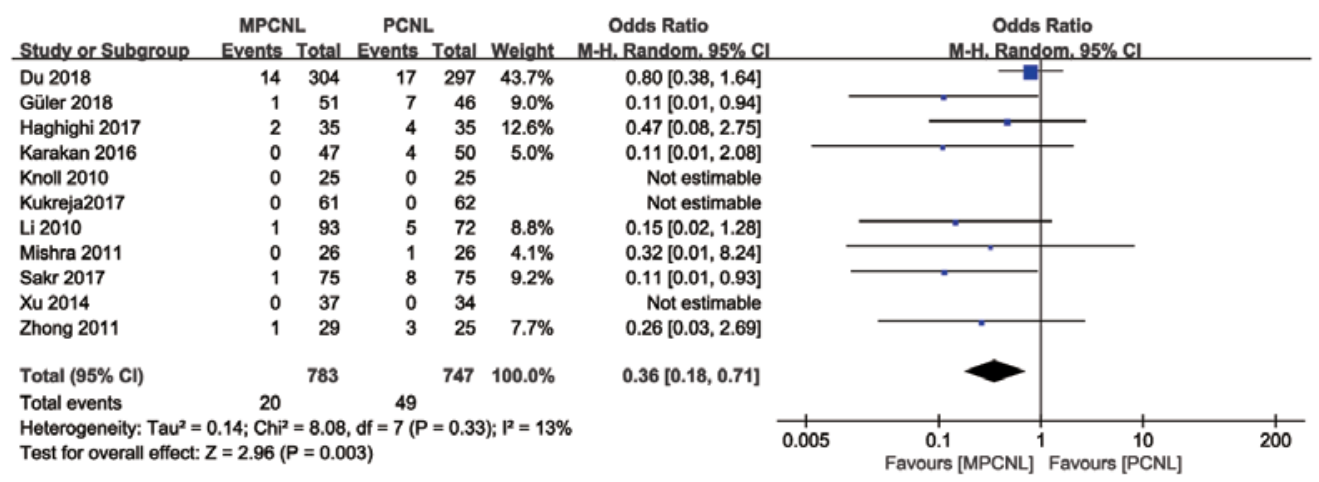

Figure 7. Forest plot of blood transfusion. Black diamonds indicate study weight. Blue squares indicate overall result. Horizontal lines indicate the $95 \%$ confidence interval. CI, confidence interval; df, degrees of freedom; M-H, Mantel-Haenszel; Random, random effects model; SD, standard deviation; PCNL, percutaneous nephrolithotomy; MCPNL, minimally invasive PCNL.

significant difference in the SFR between the MPCNL and PCNL groups, indicating that MPCNL is an effective method for treating renal stones. Numerous studies have shown the different effectiveness of PCNL and MPCNL for the treatment of renal stones $(21,41,42)$. However, the conclusion of each research may have varied due to the following factors. First, 


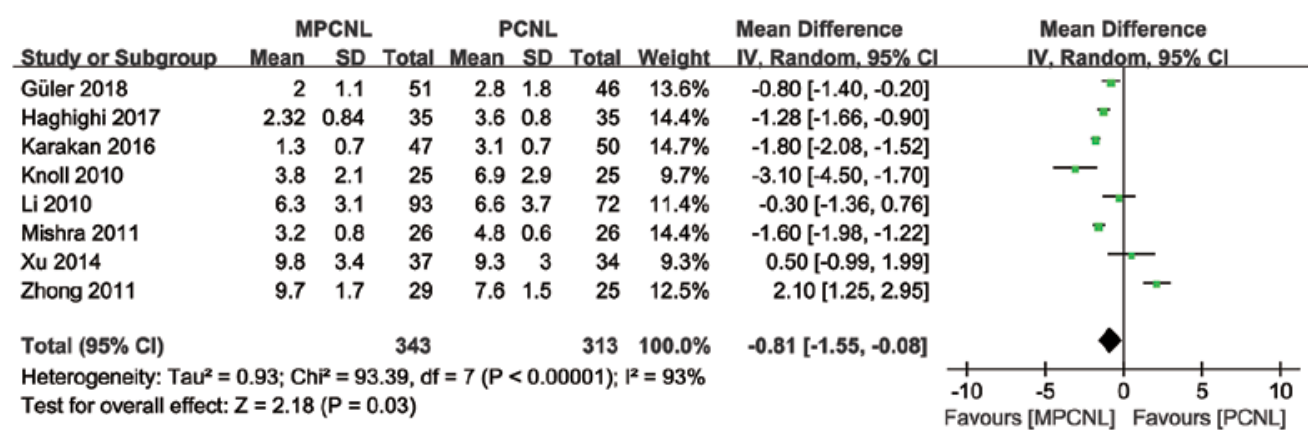

Figure 8. Forest plot of hospitalization times. Black diamonds indicate study weight. Blue squares indicate overall result. Horizontal lines indicate the 95\% confidence interval. CI, confidence interval; df, degrees of freedom; Random, random effects model; SD, standard deviation; PCNL, percutaneous nephrolithotomy; MCPNL, minimally invasive PCNL; IV, inverse variance.

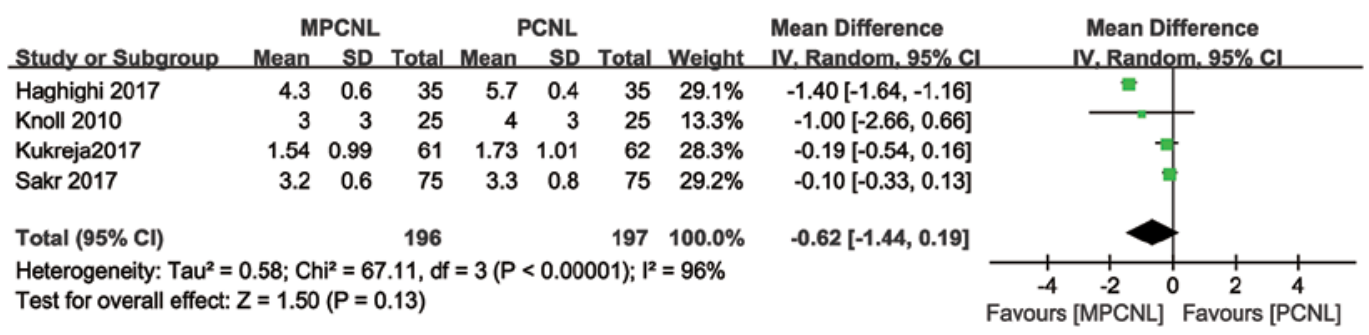

Figure 9. Forest plot of postoperative visual analogue scale. Black diamonds indicate study weight. Blue squares indicate overall result. Horizontal lines indicate the $95 \%$ confidence interval. CI, confidence interval; df, degrees of freedom; Random, random effects model; SD, standard deviation; PCNL, percutaneous nephrolithotomy; MCPNL, minimally invasive PCNL; IV, inverse variance.

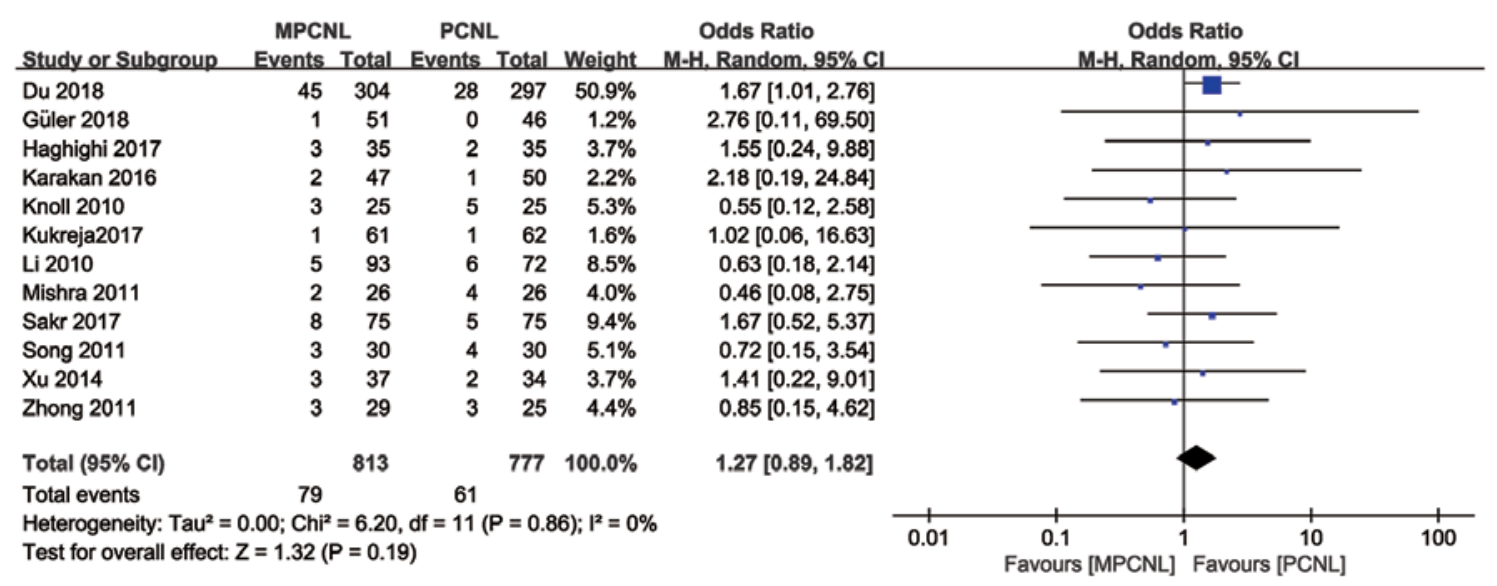

Figure 10. Forest plot of postoperative fever. Black diamonds indicate study weight. Blue squares indicate overall result. Horizontal lines indicate the $95 \%$ confidence interval. CI, confidence interval; df, degrees of freedom; M-H, Mantel-Haenszel; Random, random effects model; SD, standard deviation; PCNL, percutaneous nephrolithotomy; MCPNL, minimally invasive PCNL.

different definitions and imaging modalities were adopted to evaluate the SFR among the 12 studies. For example, imaging modalities that included plain-film X-ray and/or ultrasonography and/or non-contrast computed tomography (CT) were used to assess whether patients were free of stones. Although CT is the most accurate method to assess the SFR, it is associated with higher costs and additional radiation exposure compared to other imaging techniques (43). It is worth noting that there is no consensus on a clear definition of the SFR, which was defined as residual debris (0-4 mm) (44,45). A smaller tract may hinder the required instrumental manipulations for stone removal, especially with large calculi. The SFR is also related to the follow-up time, as the SFR 1 month after surgery is higher compared with 1 day after surgery. Time is needed for stone fragments to be flushed out with urine. The size and location of the stone also makes a difference. Some large stones even need a second round of treatment to completely remove the stone. Many urologists attempted multitract access in PCNL to elevate the SFR and achieve good clinical results. Some studies have concluded that although increasing the number of tracts exponentially increases blood loss, it does not increase the overall complication rate $(46,47)$. Lithotripsy instruments that are currently used include pneumatic and laser or ultrasonic devices and the stone could be completely 


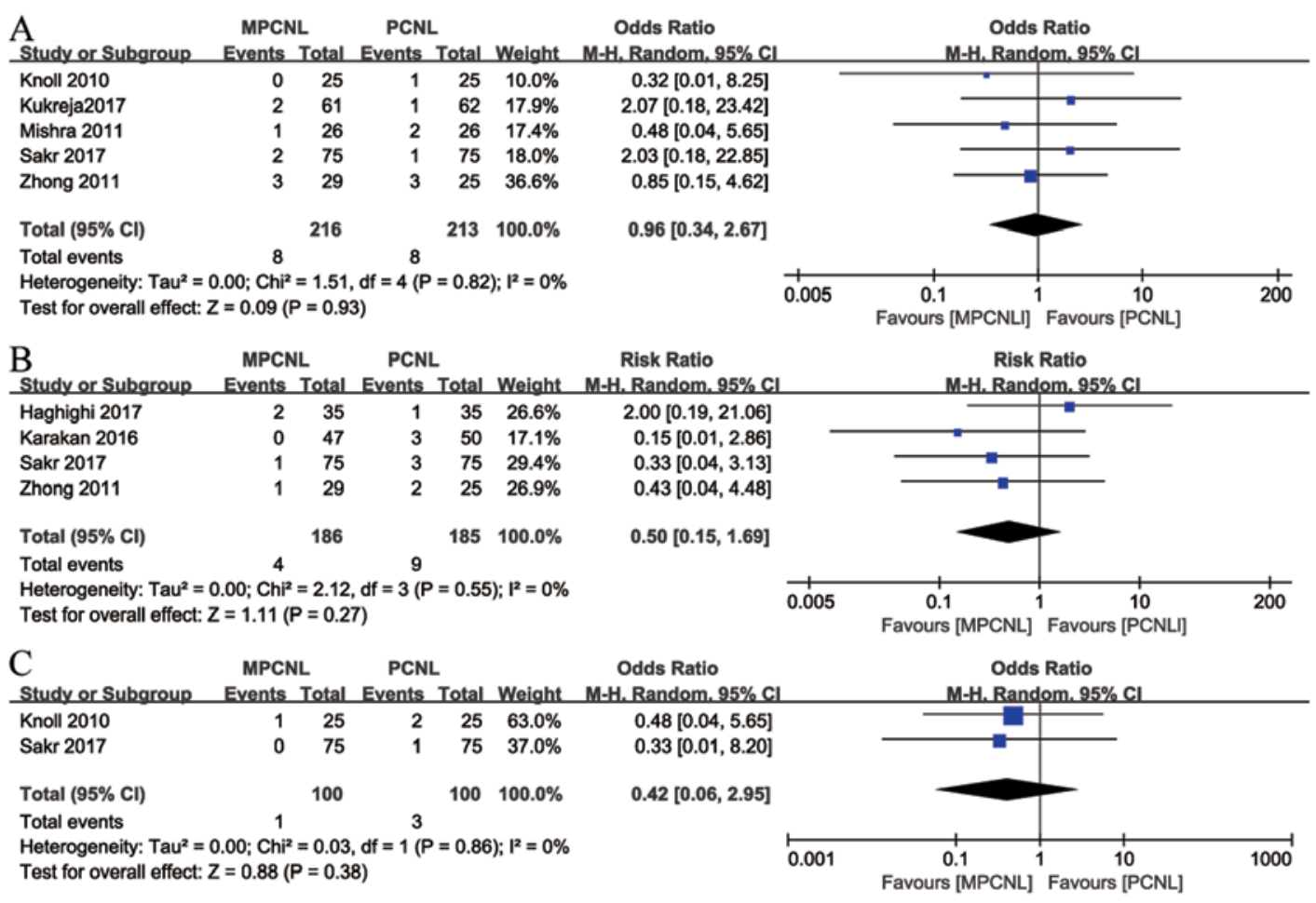

Figure 11. Forest plots of postoperative complications. (A) Urinary perforation. (B) Urinary leakage. (C) Delayed hemorrhage. Black diamonds indicate study weight. Blue squares indicate overall result. Horizontal lines indicate the $95 \%$ confidence interval. CI, confidence interval; df, degrees of freedom; M-H, Mantel-Haenszel; Random, random effects model; SD, standard deviation; PCNL, percutaneous nephrolithotomy; MCPNL, minimally invasive PCNL.

crushed when laser instruments are used, leading to a higher early SFR compared with other methods (48). The articles included in the present study used different types of lithotripsy, which influences the SFR. Clinical evaluation demonstrated that the combined usage of pneumatic and ultrasonic devices has significantly increased the efficiency of stone fragmentation $(49,50)$. More subgroups divided based on the lithotripsy methods could be analyzed to reduce bias. The included study period is between 2005-2017, during which surgical equipment and methods have significantly improved. The lithotripsy stone system is more efficient and has a better SFR compared with the ultrasound, which also offers outcome bias.

Operative times were reported in 12 of the included studies and five studies indicated that MPCNL had significantly longer operating times compared PCNL. As a result of the smaller channel and diminished intraoperative field visibility with miniature endoscopes, it is necessary to break the stones into smaller fragments to remove them, which increases the operation time of MPCNL (51). Other factors that significantly affected the operative time in the multivariate analysis were the presence of multiple stones, branching stones or multiple tracts $(52,53)$. With larger tracts, standard PCNL offers a wider working channel that bigger stone fragments could be removed by using forceps or basket. However, for MPCNL, the stone needs to be crushed into smaller pieces for the stone can be sucked out or clamped out, thus prolonging the surgical time. We hypothesize that mastering the proper technique of stone fragmentation and retrieval helps reduce the operative time and the chances of fragment migration (25). Moreover, MPCNL is more complex than PCNL and it takes more time to assemble the equipment. The operative time was not clearly defined and did not have any uniform standards, which was the largest source of heterogeneity and bias.

Regarding the staghorn stones, two studies were included in the meta-analysis. Staghorn stones still represent an intractable challenge to urologists. In the current American Urological Association guidelines, PCNL is recommended as the first-line therapy for staghorn calculi (54). The present study showed no significant difference in the SFR between the MPCNL and PCNL groups. This may be related to the use of multiple mini tracts in the MPCNL, which promoted the clearance rate. However, the operation time in MPCNL group was higher compared with PCNL. Due to due to the reduced diameter of the tract, the pressure in the pelvicaliceal system has a corresponding increase (12). The higher renal pelvic pressure (RPP) could cause high-pressure-induced bacterial endotoxin absorbance in the renal pelvis or perfusion (26). The main factor determining the RPP during the operation is the irrigation outflow (55). Xu et al (12) found that a trend towards metabolic acidosis is obvious as the irrigation time goes by. Therefore, the impact of prolonged operation time should be noted when choosing MPCNL, especially for the management of staghorn stones. For a more solid conclusion, more studies are required to verify the safety of the MPCNL in the management of staghorn stones.

A meta-analysis of complications in MPCNL and PCNL, including fever, urinary tract perforation, leakage and blood loss, was performed. The evidence showed that there were no statistically significant differences in the incidence of these complications between the two groups. Theoretically, most MPCNL technology is tubeless or use smaller nephrostomy tubes, which increased the incidence rate of urinary leakage. However, this was not proven to be the case in the 
Table III. Sensitivity analysis results.

\begin{tabular}{|c|c|c|c|c|c|c|c|c|c|}
\hline \multirow[b]{2}{*}{ Outcome } & \multirow[b]{2}{*}{ No. of studies } & \multicolumn{2}{|c|}{ Sample size (n) } & \multicolumn{4}{|c|}{ Heterogeneity (total) } & \multirow[b]{2}{*}{ MD or RR $(95 \% \mathrm{CI})$} & \multirow[b]{2}{*}{ P-value (total) } \\
\hline & & MPCNL & PCNL & $\chi^{2}$ & df & $\mathrm{I}^{2} \%$ & P-value & & \\
\hline Overall SFR & 7 & 583 & 564 & 8.82 & 6 & 32 & 0.18 & $1.02(0.96,1.08)$ & 0.55 \\
\hline Operation time & 7 & 583 & 564 & 159.31 & 6 & 96 & $<0.00001$ & $5.73(-3.86,15.31)$ & 0.24 \\
\hline Hemoglobin drop & 4 & 190 & 181 & 129.57 & 3 & 98 & $<0.00001$ & $-0.87(-1.73,-0.01)$ & 0.05 \\
\hline Hospital stay & 4 & 162 & 156 & 75.97 & 3 & 96 & $<0.00001$ & $-0.51(-1.67,0.64)$ & 0.39 \\
\hline Postoperative VAS & 2 & 110 & 110 & 59.95 & 1 & 98 & $<0.00001$ & $-0.75(-2.02,0.52)$ & 0.25 \\
\hline
\end{tabular}

CI, confidence interval; MD, mean difference; RR, risk ratio; SFR, stone-free rate; PCNL, percutaneous nephrolithotomy; MCPNL, minimally invasive PCNL; VAS, visual analogue scale; df, degree of freedom.

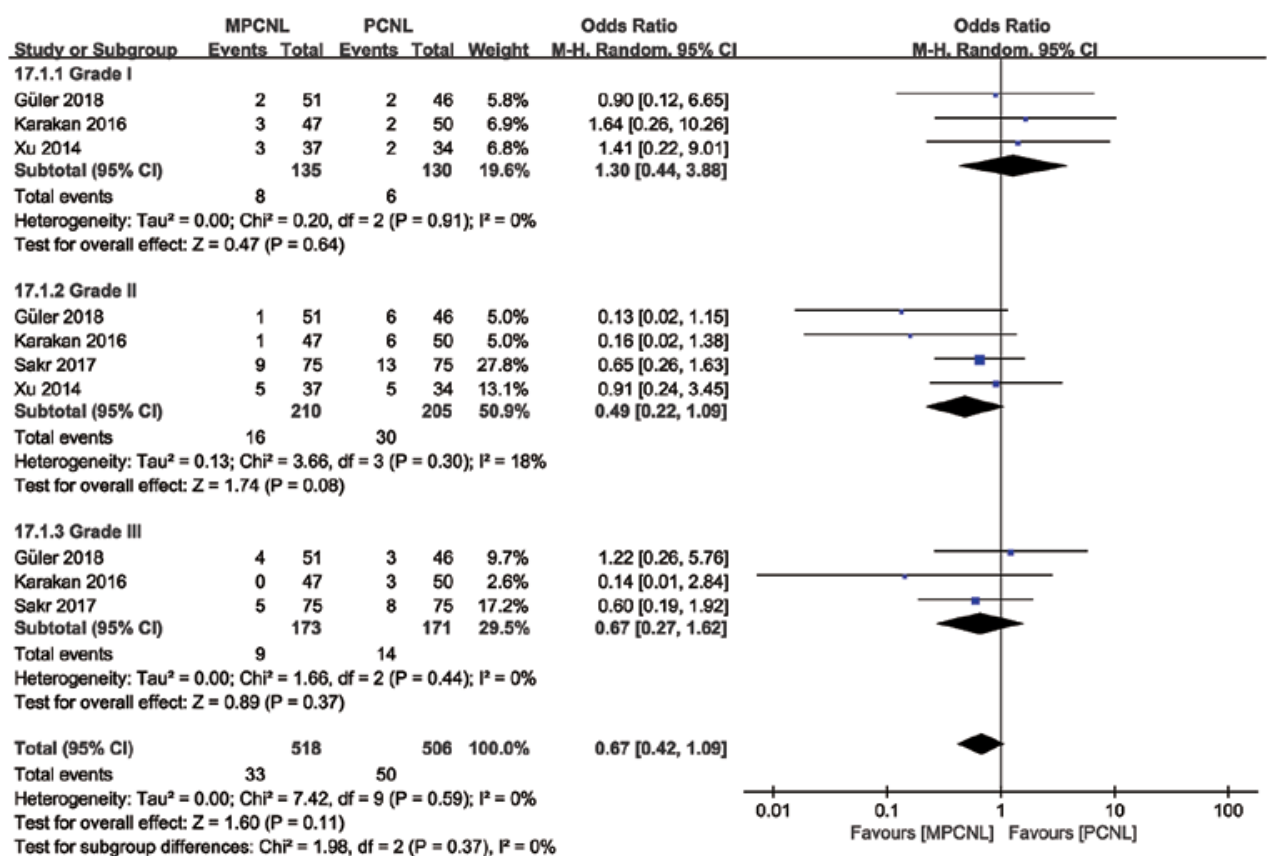

Figure 12. Forest plot of complications based on Clavien-Dindo grade system. Black diamonds indicate study weight. Blue squares indicate overall result. Horizontal lines indicate the $95 \%$ confidence interval. CI, confidence interval; df, degrees of freedom; M-H, Mantel-Haenszel; Random, random effects model; SD, standard deviation; PCNL, percutaneous nephrolithotomy; MCPNL, minimally invasive PCNL.

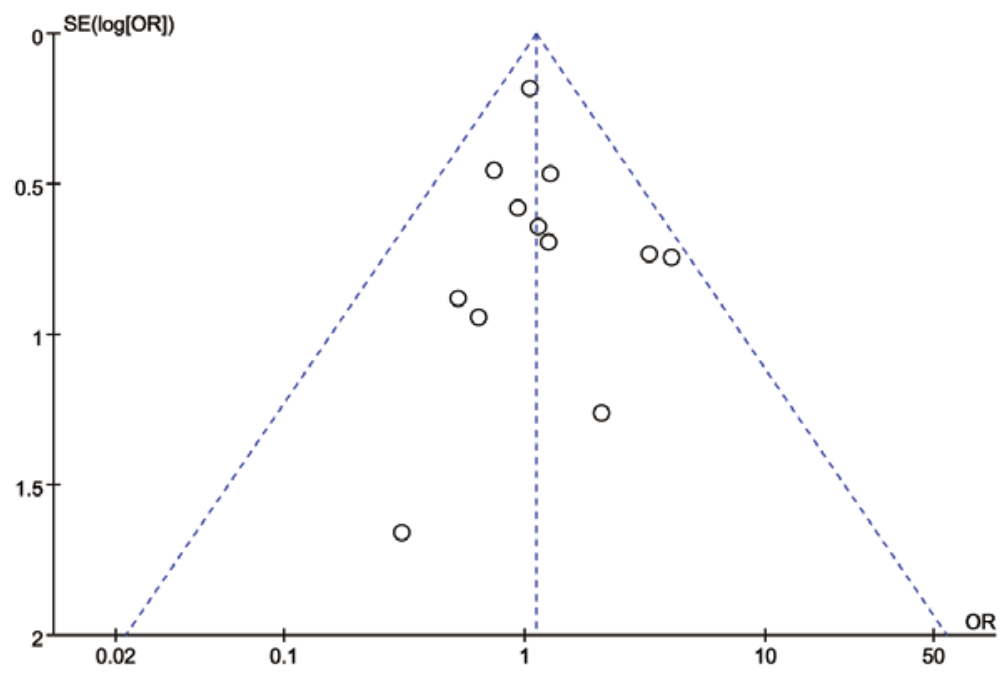

Figure 13. Funnel plot of the overall stone-free rate for publication bias. OR, odds ratio; SE, standard error. 
meta-analysis. Furthermore, a subgroup meta-analysis of postoperative complications was performed, grouping patients into grades I, II and III according to the Clavien-Dindo Classification (32). Although there were no significant differences in the Clavien-Dindo Classification complications with grades I, II and III between the two groups, the incidence of Clavien-Dindo grade II complications in the PCNL group were higher compared with the MPCNL group. From the articles that were included, the Clavien-Dindo grade II complications included pyrexia, blood transfusion, nephrostomy drainage and urinary retention. MPCNL may be safer for moderate to severe disease after surgery and the incidence of mild complications is similar between MPCNL and PCNL, showing that MPCNL has more advantages in postoperative management and may be a good choice for patients with poor basic conditions.

The main risk of standard PCNL surgery is bleeding, which may require blood transfusions and increase the risk of kidney damage (21). The important factors correlated with hemorrhage during PCNL are the larger sheath tract access, prolonged operative time, greater stone burden and multiple channels $(56,57)$. Hemorrhage is generally associated with the initial puncture and injury of renal blood vessels and the surrounding organs (27). A larger tract during PCNL may increase the risk of injury to major blood vessels and may make it more difficult to recover from puncture injury (10). Therefore, it seems that MPCNL with a smaller percutaneous channel has the potential advantage of reducing the risk of bleeding and trauma to the renal parenchyma. In the meta-analysis, previously published results confirmed the reduction in blood loss associated with MPCNL. In accordance with the lower blood loss in the MPCNL group, the rate of bleeding necessitating transfusion in the MPCNL group was significantly lower compared with the PCNL group. It is worth mentioning that delayed hemorrhage is one of the most important events requiring careful observation and management after PCNL (58). The result showed that no significant difference between MPCNL and PCNL based on the only two available studies. However, a conclusion cannot be reached owing to the limited number of RCTs. Accordingly, more studies are needed to obtain more reliable outcomes.

Theoretically, a smaller tract diameter is less invasive, which is related to a shorter hospital stay and a faster return to normal daily activities This finding was in line with both the previous studies and the present analysis. The results showed a statistically significant reduction (0.8 day shorter) in hospital stay in the MPCNL group compared with the PCNL group. Smaller tract access caused less trauma and pain. A previous study found that tubeless procedures were associated with the least postoperative pain and directly impacted the length of stay (22). However, the present results showed that no significant difference in the VAS was found between the groups. Giusti et al (13) showed no statistically significant differences in the VAS between PCNL and MPCNL. However, the VAS score in the tubeless PCNL group was lower compared with the PCNL group. It was hypothesized that pain may be associated with the presence of renal fistula drainage tubes and not the size of the tract. Several articles that were included did not indicate whether tubeless PCNL was used; this factor also has an impact on pain, which may lead to conclusion bias.

Although there have been similar studies in the past, these studies included fewer trials $(40,59)$. The present meta-analysis, which was performed using currently available comparative trials, has several limitations. A total of five prospective cohort studies were eventually included in the analysis and the included RCTs did not describe the blinding procedures clearly and in detail, which might lead to conclusion bias. Although a sensitivity or subgroup analysis was performed to show that the results were relatively stable, there is still some bias in the present conclusions caused by non-RCTs. Additionally, the heterogeneity among these trials was found to be high in terms of several parameters. The heterogeneity in these parameters can be explained by the difference in outcome definitions, measurement and standards, such as for the residual stone fragments and postoperative VAS. Furthermore, the calculus size, calculus location and surgical equipment varied in each evaluated study. Besides, there were no uniform inclusion criteria in the included studies. The results should be interpreted with caution due to a mixed group of patients. In addition, the availability of technical equipment and surgical experience also plays a critical role in evaluating the effectiveness of both techniques and could not be assessed in the present review. However, the present study provided the most updated information about the comparison of MPCNL and PCNL for the treatment of renal stones. Finally, only a small number of studies were identified through the systematic review of the literature and were then enrolled in the meta-analysis. More high-quality RCTs are needed to further evaluate the effectiveness and safety of these two surgical techniques.

To conclude, the meta-analysis indicated that MPCNL is an effective method for treating renal stones. Compared with PCNL, it not only had similar high cure rates, but also was associated with significantly less bleeding and a lower transfusion rate. However, further larger, well-designed prospective RCTs with a larger patient series are required to confirm this conclusion.

\section{Acknowledgements}

Not applicable.

\section{Funding}

No funding was received.

\section{Availability of data and materials}

The datasets used and/or analyzed during the current study are available from the corresponding author on reasonable request.

\section{Authors' contributions}

GZ, JY, BJ and ZL designed the study. BJ and ZL collected data. BJ, ZL and TH analyzed and interpreted data. BJ and ZL wrote the manuscript. All authors read and approved the final manuscript.

\section{Ethics approval and consent to participate}

Not Applicable. 


\section{Patient consent for publication}

Not applicable.

\section{Competing interests}

The authors declare that they have no competing interests.

\section{References}

1. Dwyer ME, Krambeck AE, Bergstralh EJ, Milliner DS, Lieske JC and Rule AD: Temporal trends in incidence of kidney stones among children: A 25-year population based study. J Urol 188: $247-252,2012$

2. Marickar YM and Vijay A: Female stone disease: The changing trend. Urol Res 37: 337-340, 2009.

3. Turk C, Knoll T, Petrik A, Sarica K, Skolarikos A, Straub M and Seitz C: Guidelines on urolithiasis. European Association of Urology, 2015. http://uroweb.org/wp-content/uploads/22-Urolithiasis_LR_full.pdf.

4. De la Rosette JJ, Opondo D, Daels FP, Giusti G, Serrano A Kandasami SV, Wolf JS Jr, Grabe M and Gravas S; CROES PCNL Study Group: Categorisation of complications and validation of the Clavien score for percutaneous nephrolithotomy. Eur Urol 62: 246-255, 2012.

5. Cicekbilek I, Resorlu B, Oguz U, Kara C and Unsal A: Effect of percutaneous nephrolithotomy on renal functions in children: Assessment by quantitative SPECT of (99 m)Tc-DMSA uptake by the kidneys. Ren Fail 37: 1118-1121, 2015.

6. Karatag T, Tepeler A, Silay MS, Bodakci MN, Buldu I, Daggulli M, Hatipoglu NK, Istanbulluoglu MO and Armagan A A comparison of 2 percutaneous nephrolithotomy techniques for the treatment of pediatric kidney stones of sizes $10-20 \mathrm{~mm}$ : Microperc vs Miniperc. Urology 85: 1015-1018, 2015.

7. Jackman SV, Hedican SP, Peters CA and Docimo SG: Percutaneous nephrolithotomy in infants and preschool age children: Experience with a new technique. Urology 52: 697-701, 1998.

8. Lahme S, Bichler KH, Strohmaier WL and Götz T: Minimally invasive PCNL in patients with renal pelvic and calyceal stones Eur Urol 40: 619-624, 2001.

9. Zeng G, Zhu W and Lam W: Miniaturised percutaneous nephrolithotomy: Its role in the treatment of urolithiasis and our experience. Asian J Urol 5: 295-302, 2018.

10. Hennessey DB, Kinnear NK, Troy A, Angus D, Bolton DM and Webb DR: Mini PCNL for renal calculi: Does size matter. BJU Int 119 (Suppl 5): S39-S46, 2017.

11. Abdelhafez MF, Wendt-Nordahl G, Kruck S, Mager R, Stenzl A, Knoll T and Schilling D: Minimally invasive versus conventional large-bore percutaneous nephrolithotomy in the treatment of large-sized renal calculi: Surgeon's preference. Scand J Urol 50: 212-215, 2016

12. Xu S, Shi H, Zhu J, Wang Y, Cao Y, Li K, Wang Y, Sun Z and Xia S: A prospective comparative study of haemodynamic, electrolyte, and metabolic changes during percutaneous nephrolithotomy and minimally invasive percutaneous nephrolithotomy. World J Urol 32: 1275-1280, 2014.

13. Giusti G, Piccinelli A, Taverna G, Benetti A, Pasini L, Corinti M, Teppa A, Zandegiacomo de Zorzi S and Graziotti P: Miniperc? No, thank you. Eur Urol 51: 810-815, 2007.

14. Moher D, Liberati A, Tetzlaff J and Altman DG; PRISMA Group: Preferred reporting items for systematic reviews and meta-analyses: The PRISMA statement. PLoS Med 6: e1000097, 2009.

15. Shea BJ, Reeves BC, Wells G, Thuku M, Hamel C, Moran J, Moher D, Tugwell P, Welch V, Kristjansson E and Henry DA: AMSTAR 2: A critical appraisal tool for systematic reviews that include randomised or non-randomised studies of healthcare interventions, or both. BMJ 358: j4008, 2017.

16. Mostafa A, Agur W, Abdel-All M, et al: Oxford Centre for Evidence-based Medicine-Levels of Evidence. 2009. http://www.cebm.net/ox ford-Centre-evidence-based-medicine levels-evidencemarch-2009/. Accessed May, 2016.

17. Clark HD, Wells GA, Huët C, McAlister FA, Salmi LR, Fergusson D and Laupacis A: Assessing the quality of randomized trials: Reliability of the Jadad scale. Control Clin Trials 20: 448-452, 1999.
18. Wells GA, Shea B, O'Connell, Robertson J, Peterson J, Welch V, Losos M and Tugwell P: The Newcastle-Ottawa Scale (NOS) for Assessing the Quality if Nonrandomized Studies in Meta-Analyses. Ottawa Hospital Research Institute, 2015. http://www.ohri.ca/programs/clinical_epidemiology/oxford.asp. Accessed May 2016.

19. Higgins JP, Altman DG, Gøtzsche PC, Jüni P, Moher D, Oxman AD, Savovic J, Schulz KF, Weeks L, Sterne JA, et al: The Cochrane collaboration's tool for assessing risk of bias in randomised trials. BMJ 343: d5928, 2011.

20. Du C, Song L, Wu X, Fan D, Zhu L, Liu S, Deng X, Liu T, Yang Z, Peng $\mathrm{Z}$, et al: Suctioning minimally invasive percutaneous nephrolithotomy with a patented system is effective to treat renal staghorn calculi: A prospective multicenter study. Urol Int 101: 143-149, 2018.

21. Güler A, Erbin A,Ucpinar B, Savun M, Sarilar O and Akbulut MF: Comparison of miniaturized percutaneous nephrolithotomy and standard percutaneous nephrolithotomy for the treatment of large kidney stones: A randomized prospective study. Urolithiasis 47: 289-295, 2019.

22. Haghighi R, Zeraati $H$ and Ghorban Zade M: Ultra-minipercutaneous nephrolithotomy (PCNL) versus standard PCNL: A randomised clinical trial. Arab J Urol 15: 294-298, 2017.

23. Karakan T, Kilinc MF, Doluoglu OG, Yildiz Y, Yuceturk CN, Bagcioglu M, Karagöz MA, Bas O and Resorlu B: The modified ultra-mini percutaneous nephrolithotomy technique and comparison with standard nephrolithotomy: A randomized prospective study. Urolithiasis 45: 209-213, 2017.

24. Knoll T, Wezel F, Michel MS, Honeck P and Wendt-Nordahl G: Do patients benefit from miniaturized tubeless percutaneous nephrolithotomy? A comparative prospective study. J Endourol 24: 1075-1079, 2010.

25. Kukreja RA: Should mini percutaneous nephrolithotomy (MiniPNL/Miniperc) be the ideal tract for medium-sized renal calculi (15-30 mm)? World J Urol 36: 285-291, 2018.

26. Li LY, Gao X, Yang M, Li JF, Zhang HB, Xu WF and Lin Z: Does a smaller tract in percutaneous nephrolithotomy contribute to less invasiveness? A prospective comparative study. Urology 75: 56-61, 2010.

27. Mishra S, Sharma R, Garg C, Kurien A, Sabnis R and Desai M: Prospective comparative study of miniperc and standard PNL for treatment of 1 to $2 \mathrm{~cm}$ size renal stone. BJU Int 108: 896-900, 2011.

28. Sakr A, Salem E, Kamel M, Desoky E, Ragab A, Omran M, Fawzi A and Shahin A: Minimally invasive percutaneous nephrolithotomy vs standard PCNL for management of renal stones in the flank-free modified supine position: Single-center experience. Urolithiasis 45: 585-589, 2017.

29. Song L, Chen Z, Liu T, Zhong J, Qin W, Guo S, Peng Z, Hu M, Du C, Zhu L, et al: The application of a patented system to minimally invasive percutaneous nephrolithotomy. J Endourol 25: 1281-1286, 2011.

30. Zhong W, Zeng G, Wu W, Chen W and Wu K: Minimally invasive percutaneous nephrolithotomy with multiple mini tracts in a single session in treating staghorn calculi. Urol Res 39: 117-122, 2011.

31. Hozo SP, Djulbegovic B and Hozo I: Estimating the mean and variance from the median, range, and the size of a sample. BMC Med Res Methodol 5: 13, 2005 .

32. Tefekli A, Ali Karadag M, Tepeler K, Sari E, Berberoglu Y, Baykal M, Sarilar O and Muslumanoglu AY: Classification of percutaneous nephrolithotomy complications using the modified Clavien grading system: Looking for a standard. Eur Urol 53: 184-190, 2008

33. Moe OW: Kidney stones: Pathophysiology and medical management. Lancet 367: 333-344, 2006.

34. Assimos D, Krambeck A, Miller NL, Monga M, Murad MH, Nelson CP, Pace KT, Pais VM Jr, Pearle MS, Preminger GM, et al: Surgical management of stones: American Urological Association/Endourological Society Guideline, PART II J Urol 196: 1161-1169, 2016.

35. De la Rosette J, Assimos D, Desai M, Gutierrez J, Lingeman J, Scarpa R and Tefekli A; CROES PCNL Study Group: The clinical research office of the endourological society percutaneous nephrolithotomy global study: Indications, complications, and outcomes in 5803 patients. J Endourol 25: 11-17, 2011.

36. Kirac M, Bozkurt ÖF, Tunc L, Guneri C, Unsal A and Biri H: Comparison of retrograde intrarenal surgery and mini-percutaneous nephrolithotomy in management of lower-pole renal stones with a diameter of smaller than $15 \mathrm{~mm}$. Urolithiasis 41: 241-246, 2013. 
37. Sabnis RB, Ganesamoni R and Sarpal R: Miniperc: What is its current status? Curr Opin Urol 22: 129-133, 2012.

38. Desai MR, Sharma R, Mishra S, Sabnis RB, Stief C and Bader M: Single-step percutaneous nephrolithotomy (microperc): The initial clinical report. J Urol 186: 140-145, 2011.

39. De S, Autorino R, Kim FJ, Zargar H, Laydner H, Balsamo R, Torricelli FC, Di Palma C, Molina WR, Monga M and De Sio M: Percutaneous nephrolithotomy versus retrograde intrarenal surgery: A systematic review and meta-analysis. Eur Urol 67: 125-137, 2015.

40. Zhu W, Liu Y, Liu L, Lei M, Yuan J, Wan SP and Zeng G: Minimally invasive versus standard percutaneous nephrolithotomy: A meta-analysis. Urolithiasis 43: 563-570, 2015.

41. Mahmood SN, Aziz BO, Tawfeeq HM and Fakhralddin SS Mini-versus standard percutaneous nephrolithotomy for treatment of pediatric renal stones: Is smaller enough. J Pediatr Urol 15: 664.e1-664.e6, 2019.

42. Wu C, Hua LX, Zhang JZ, Zhou XR, Zhong W and Ni HD Comparison of renal pelvic pressure and postoperative fever incidence between standard- and mini-tract percutaneous nephrolithotomy. Kaohsiung J Med Sci 33: 36-43, 2017.

43. Davis NF, Quinlan MR, Poyet C, Lawrentschuk N, Bolton DM, Webb D and Jack GS: Miniaturised percutaneous nephrolithotomy versus flexible ureteropyeloscopy: A systematic review and meta-analysis comparing clinical efficacy and safety profile. World J Urol 36: 1127-1138, 2018.

44. Somani BK, Desai M, Traxer O and Lahme S: Stone-free rate (SFR): A new proposal for defining levels of SFR. Urolithiasis 42 : 95, 2014.

45. Danilovic A, Cavalanti A, Rocha BA, Traxer O, Torricelli FCM Marchini GS, Mazzucchi E and Srougi M: Assessment of residual stone fragments after retrograde intrarenal surgery. J Endourol 32: 1108-1113, 2018.

46. Manohar T, Ganpule AP, Shrivastav P and Desai M: Percutaneous nephrolithotomy for complex caliceal calculi and staghorn stones in children less than 5 years of age. J Endourol 20: 547-551, 2006

47. Cho HJ, Lee JY, Kim SW, Hwang TK and Hong SH: Percutaneous nephrolithotomy for complex renal calculi: Is multi-tract approach ok? Can J Urol 19: 6360-6365, 2012.

48. Chen S, Zhou L, Wei T, Luo D, Jin T, Li H and Wang K: Comparison of holmium: YAG laser and pneumatic lithotripsy in the treatment of ureteral stones: An update meta-analysis. Urol Int 98: 125-133, 2017.

49. Hofmann R, Weber J, Heidenreich A, Varga Z and Olbert P. Experimental studies and first clinical experience with a new Lithoclast and ultrasound combination for lithotripsy. Eur Urol 42: 376-381, 2002.

50. Kuo RL, Paterson RF, Siqueira TM Jr, Evan AP, McAteer JA, Williams JC Jr and Lingeman JE: In vitro assessment of lithoclast ultra intracorporeal lithotripter. J Endourol 18: 153-156, 2004 .
51. Ergin G, Kirac M, Kopru B, Ebiloglu T and Biri H: Flexible ureterorenoscopy versus mini-percutaneous nephrolithotomy for the treatment of renal stones. Urol J 15: 313-317, 2018.

52. ElSheemy MS, Elmarakbi AA, Hytham M, Ibrahim H, Khadgi S and Al-Kandari AM: Mini vs standard percutaneous nephrolithotomy for renal stones: A comparative study. Urolithiasis 47 207-214, 2019.

53. Akman T, Sari E, Binbay M, Yuruk E, Tepeler A, Kaba M, Muslumanoglu AY and Tefekli A: Comparison of outcomes after percutaneous nephrolithotomy of staghorn calculi in those with single and multiple accesses. J Endourol 24: 955-960, 2010.

54. Preminger GM, Assimos DG, Lingeman JE, Nakada SY, Pearle MS and Wolf JS Jr; AUA Nephrolithiasis Guideline Panel: Chapter 1: AUA guideline on management of staghorn calculi: Diagnosis and treatment recommendations. J Urol 173 : 1991-2000, 2005.

55. Tokas T, Herrmann TRW, Skolarikos A and Nagele U; Training and Research in Urological Surgery and Technology (T.R.U.S.T.)-Group: Pressure matters: Intrarenal pressures during normal and pathological conditions, and impact of increased values to renal physiology. World J Urol 37: 125-131, 2019.

56. Yamaguchi A, Skolarikos A, Buchholz NP, Chomón GB, Grasso M, Saba P, Nakada S and de la Rosette J; Clinical Research Office Of The Endourological Society Percutaneous Nephrolithotomy Study Group: Operating times and bleeding complications in percutaneous nephrolithotomy: A comparison of tract dilation methods in 5,537 patients in the clinical research office of the Endourological society percutaneous Nephrolithotomy global study. J Endourol 25: 933-939, 2011.

57. Kukreja R, Desai M, Patel S, Bapat S and Desai M: Factors affecting blood loss during percutaneous nephrolithotomy: Prospective study. J Endourol 18: 715-722, 2004.

58. Nouralizadeh A, Ziaee SA, Hosseini Sharifi SH, Basiri A, Tabibi A, Sharifiaghdas F, Zaki H, Nikkar MM, Lashay A, Ahanian A and Soltani MH: Delayed postpercutaneous nephrolithotomy hemorrhage: Prevalence, predictive factors and management. Scand J Urol 48: 110-115, 2014.

59. Gao XS, Liao BH, Chen YT, Feng SJ, Gao R, Luo DY, Liu JM and Wang KJ: Different tract sizes of miniaturized percutaneous nephrolithotomy versus retrograde intrarenal surgery: A systematic review and meta-analysis. J Endourol 31: 1101-1110, 2017.

This work is licensed under a Creative Commons Attribution-NonCommercial-NoDerivatives 4.0 International (CC BY-NC-ND 4.0) License. 